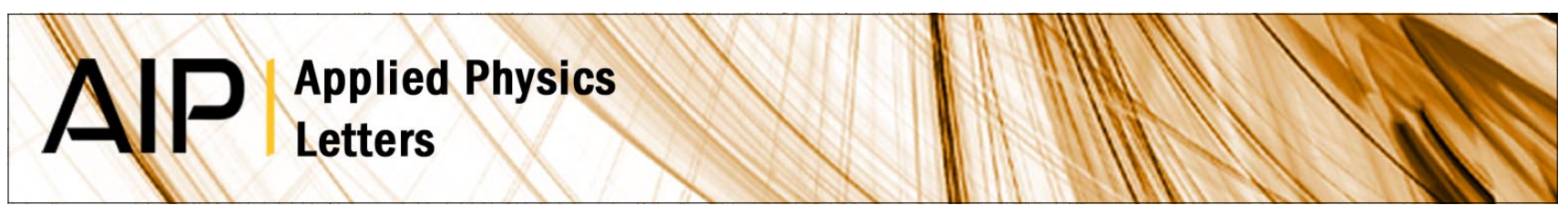

\title{
Isotopic enrichment in a plasma centrifuge
}

E. Del Bosco, R. S. Dallaqua, G. O. Ludwig, and J. A. Bittencourt

Citation: Appl. Phys. Lett. 50, 1716 (1987); doi: 10.1063/1.97725

View online: http://dx.doi.org/10.1063/1.97725

View Table of Contents: http://apl.aip.org/resource/1/APPLAB/v50/i24

Published by the American Institute of Physics.

Additional information on Appl. Phys. Lett.

Journal Homepage: http://apl.aip.org/

Journal Information: http://apl.aip.org/about/about_the_journal

Top downloads: http://apl.aip.org/features/most_downloaded

Information for Authors: http://apl.aip.org/authors

\section{ADVERTISEMENT}

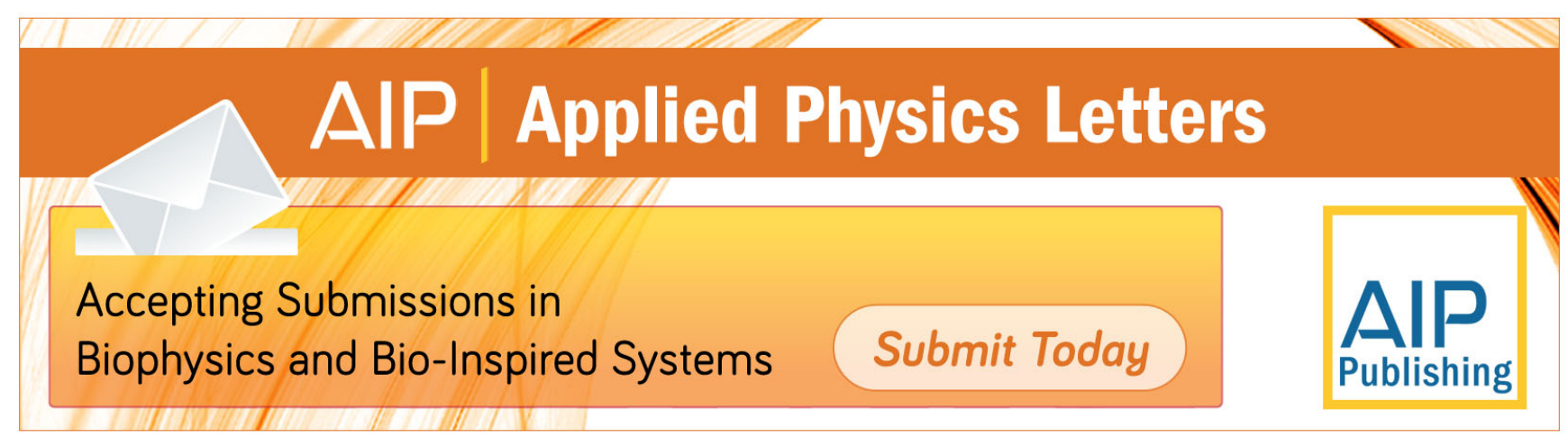




\title{
Isotopic enrichment in a plasma centrifuge
}

\author{
E. Del Bosco, R. S. Dallagua, G. O. Ludwig, and J. A. Bittencourt \\ Laboratório Associado de Plasmas, Instituto de Pesquisas Espaciais-INPE/MCT, Caixa Postal 515 , 12201 \\ Säo José dos Campos. SP, Brazil
}

(Received 5 February 1987; accepted for publication 20 April 1987)

\begin{abstract}
High rotational velocity and centrifugal isotopic separation of carbon in a vacuum-arc plasma centrifuge are presented. Enrichments of up to $390 \%$ for ${ }^{13} \mathrm{C}$ are measured at $6 \mathrm{~cm}$ radius with angular rotation frequencies in excess of $1.0 \times 10^{5} \mathrm{rad} / \mathrm{s}$ in an axial magnetic field of $0.12 \mathrm{~T}$.
\end{abstract}

The plasma centrifuge is a device in which a plasma column is made to rotate at high angular velocity by means of an electromagnetic force. Partially ionized gases and highly ionized gases with a neutral gas envelope were used in earlier experiments in this area. Bonnevier was the first to measure isotope separation in gas discharge rotating plasmas, achieving relatively low enrichment. Higher enrichments were obtained by James and Simpson ${ }^{2}$ in Ne plasmas. In these and other experiments with gas discharge centrifuges, a critical limiting velocity, known as the Alfén critical velocity, has been observed. This limiting velocity seems to be connected with the deleterious effects of the drag of neutral atoms on the rotating ions in gas discharge centrifuges, as suggested by Alfvén. ${ }^{3}$

To avoid this effect and increase the rotational velocity and the enrichment, it is necessary to produce a highly ionized plasma. Isotope separation in fully ionized carbon and metal plasmas produced by a laser-initiated vacuum are in an externally applied axial magnetic field was first reported by Krishnan et al., 4,5 where rotational velocities above the Alfvén critical velocity and high separation factors were obtained,

The separation factor $\alpha$ is usually defined as

$$
\alpha=\frac{\left(n_{1} / n_{2}\right)}{\left(n_{1} / n_{2}\right)}
$$

where $n_{1}$ and $n_{2}$ denote the number densities of any two species considered. The subscript o refers to natural abundance and $e$ to enriched abundance. For steady-state conditions and considering that the different ion species have the same temperature and charge state, the separation factor can be expressed as ${ }^{5}$

$$
\alpha=\exp \left(\Delta m \omega^{2} R^{2} / 2 k T\right),
$$

where $\Delta m$ is the mass difference of the ions, $\omega$ is the angular rotation frequency, $R$ is the radial distance from the column axis, $k$ is the Boltzmann's constant, and $T$ 'is the ion temperature.

In the present experiment, a rotating fully ionized plasma column has been produced in a vacuum-arc centrifuge. This letter describes the apparatus and shows new results for the rotational velocity and isotope enrichment of carbon and metal plasmas. The ion rotation velocity is derived from electrostatic probe measurements and from the azimuthal displacement of the material deposited behind a narrow sit. The isotope enrichment is measured with a modified quadrupole mass spectrometer, which determines, in situ, the relative abundance of the isotopes at the end of the plasma column at various radial positions.

Figure 1 shows schematically the plasma centrifuge apparatus. ${ }^{6}$ The stainless-steel vacuum vessel has a diameter of $22 \mathrm{~cm}$ and is $85 \mathrm{~cm}$ long, evacuated to $10^{-7}$ Torr by a diffusion pump. Eight magnet coils produce a peak $B$ field of 1.3 $T$, with a $185 \mathrm{~ms} L / R$ decay tine. The are is triggered by a $Q$ switched ruby laser. The discharge current is produced by $a$ low voltage overdamped RLC network, with $1-10 \mathrm{kA}$ peak value depending on the shot conditions. The main feature of the device is the high $B$ field, allowing the study of the separating factor dependence upon the magnetic field strength, over a wide range of values. The strong axial gradient in the magnetic field, decreasing to $10 \%$ of the peak strength at 50 $\mathrm{cm}$ from the cathode, and the small distance between cathode and end plate make the device significantly difierent from the YALE experiment. ${ }^{7}$

To measure the plasma rotation two experiments are performed. In the first experiment, two parallel Mylar sheets, with a small distance between them, are placed across the plasma column (axial position C in Fig. 1). After several tens of shots, with fixed conditions, a thin film composed of the cathode material is deposited on the first sheet, in which there is a narrow shit. The plasma which goes through the slit produces an azimuthally displaced image of the slit on the second sheet. Depending on the magnetic field direction, the slit image observed rotates to the right or to the left. Measuring the angle between the slit and its deposited image and assuming an axial velocity of $6 \times 10^{5}-1 \times 10^{6} \mathrm{~cm} / \mathrm{s}^{7}$ angular rotation frequency values of $6.9 \times 10^{4}-1.2 \times 10^{5} \mathrm{rad} / \mathrm{s}$ for a carbon plasma and $6.9 \times 10^{4}-1.7 \times 10^{5} \mathrm{rad} / \mathrm{s}$ for a copper plasma are measured, both with an externally applied magnetic field of $0.09 \mathrm{~T}$.

In the second experiment, two electrostatic probes are

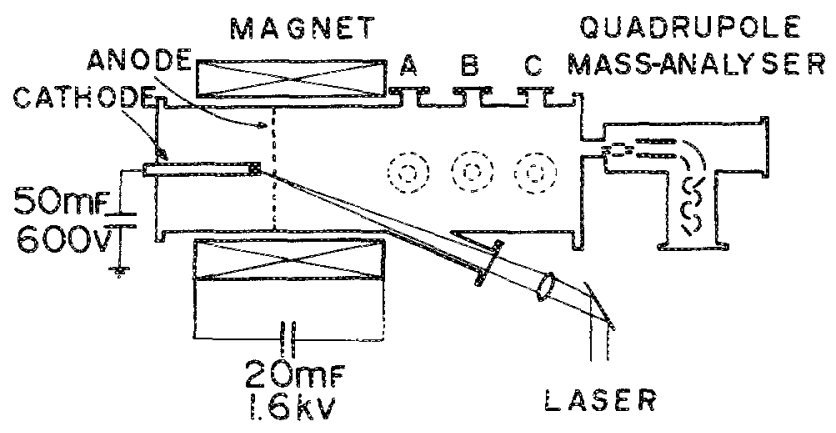

FIG. 1. Schematic diagram of INPE's vacuum-are centrifuge. 


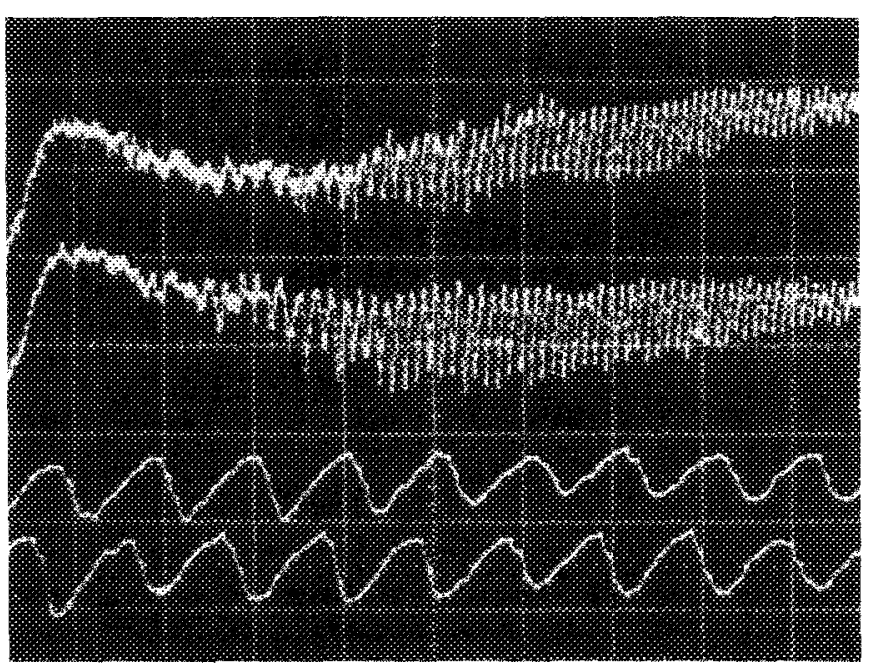

FIG. 2. Floating potential at $R=5 \mathrm{~cm}$ measured with two probes $90^{\circ}$ apart in azimuth for a carbon plasma. The ordinate scale is $2 \mathrm{~V} / \mathrm{div}$. Two upper curves: $1 \mathrm{~ms} /$ div; two lower curves: $100 \mu \mathrm{s} / \mathrm{div}$.

placed $90^{\circ}$ apart in azimuth, at $34 \mathrm{~cm}$ from the cathode. The time evolution of the plasma floating potential measured by the probes shows a characteristic periodic fluctuation, which is due to radial density inhomogeneities in the rovating plasma column. These periodic fuctuations disappear in the absence of the magnetic field. A regular fluctuation is obtained by tuning the $B$ field. Figure 2 shows the floating potential measured by two probes in a carbon plasma at $R=5 \mathrm{~cm}$ with $B=0.09 \mathrm{~T}$. The two upper curves are the complete signal, and the two lower ones are a ten times expanded view of a portion of the upper curves. An angular rotation frequency of $1.0 \times 10^{5} \mathrm{rad} / \mathrm{s}$ is obtained from the phase difference between the two probes and from the frequency of the periodic fluctuations, in the range measured by the slit method. This angular rotation frequency yields a rotational velocity of $5.0 \times 10^{5} \mathrm{~cm} / \mathrm{s}$ at $R=5 \mathrm{~cm}$, in excess of the Alfvén critical velocity of $1.34 \times 10^{5} \mathrm{~cm} / \mathrm{s}$ for carbon. A rotational velocity of $8.4 \times 10^{5} \mathrm{~cm} / \mathrm{s}$ for a magnesium plasma at $R=4$ $\mathrm{cm}$ and $B=0.1 \mathrm{~T}$, has also been measured. This value is

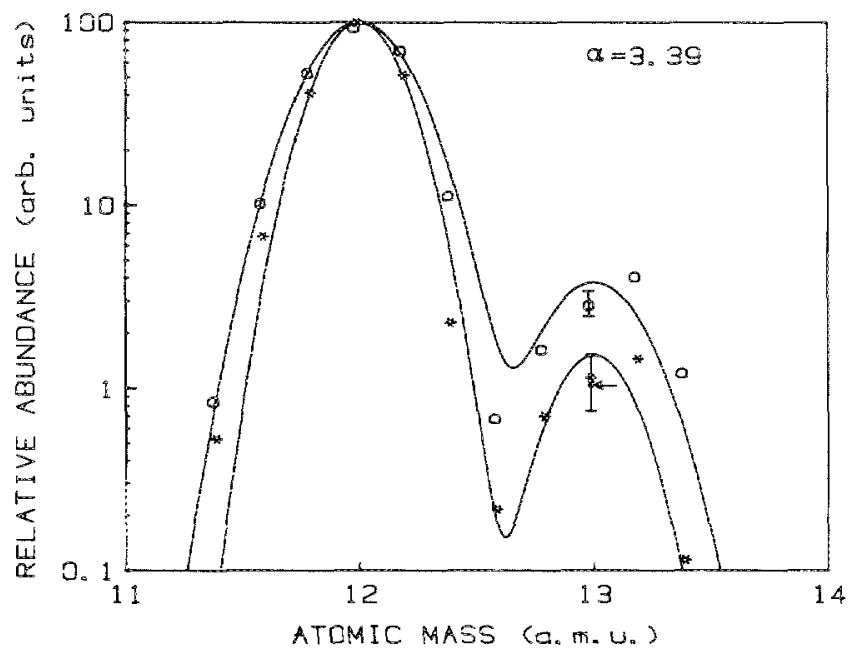

FIG. 3. Mass scan of carbon plasma in logarithmic scale. Upper curve for $B=0.12 \mathrm{~T}, I=0.8 \mathrm{kA}$ and lower curve for $B=0, I=2.3 \mathrm{kA}$, both measured at $R=6.0 \mathrm{~cm}$.

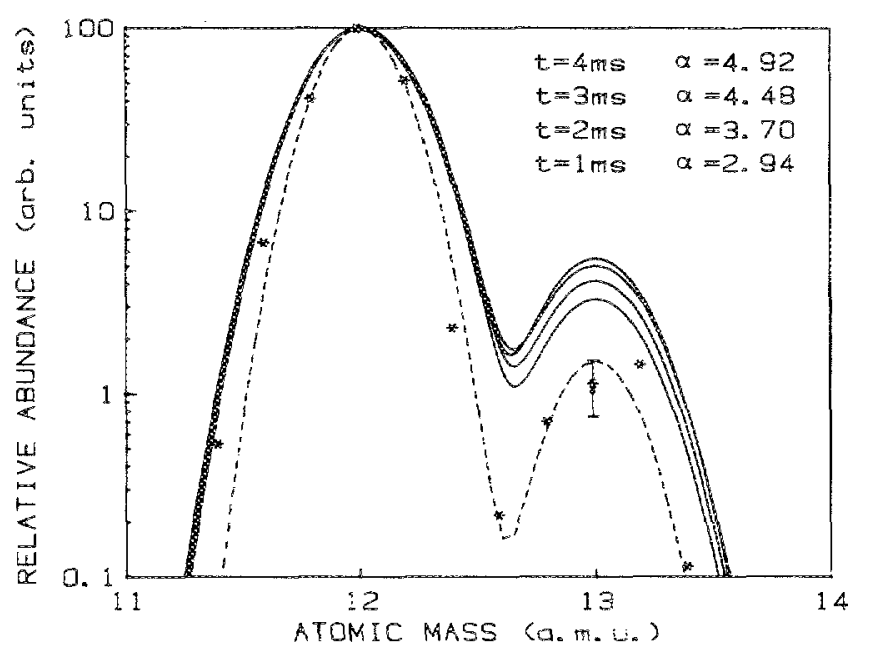

FIG. 4. Best fit of two Gaussians for data taken in four different times, in logarithmic scale, for $B=0.12 \mathrm{~T}, I=0.8 \mathrm{kA}$, and $R=6.0 \mathrm{~cm}$. The dashed curve is for $B=0, I=2.3 \mathrm{kA}$, and $R=6.0 \mathrm{~cm}$.

greater than the Alfvén critical velocity of $2.46 \times 10^{5} \mathrm{~cm} / \mathrm{s}$. Reversing the magnetic field direction, the phase difference is maintained but the rotation direction is reversed, as expected. Considering an axial velocity in the range specified previously and an angular rotation frequency of $1.0 \times 10^{5}$ $\mathrm{rad} / \mathrm{s}$, a plasma fuid element completes one to two revolutions before reaching the end plate.

The ions at the end of the plasma column are mass scanned using a quadrupole mass spectrometer. Some modifications were made in the quadrupole for proper extraction and analysis of positive ions from a plasma with a negative potential. Figure 3 shows the relative abundance of the carbon isotopes measured with the mass spectrometer and a computed best fit of two Gaussians, in logarithmic scale, for the experimental points. The lower trace is the best fit for the scan without magnetic field and the upper one for $B=0.12$ $T$, both taken at $R=6 \mathrm{~cm}$. The peaks at ${ }^{12} \mathrm{C}$ are normalized to the same value. The point marked with an arrow represents the natural abundance of ${ }^{13} \mathrm{C}$. The ratio between the adjusted ${ }^{13} \mathrm{C}$ peak and the natural value is the separation factor defined earlier, which in this case is $3.39 \pm 0.53$, implying an enrichment of $239 \%$ or, in other words, a composition of $96.34 \%{ }^{12} \mathrm{C}$ and $3.66 \%{ }^{13} \mathrm{C}$, while the natural carbon composition is $98.89 \%{ }^{12} \mathrm{C}$ and $1.11 \%{ }^{13} \mathrm{C}$.

Figure 4 shows the best fit for the time evolution of the carbon mass spectrum. The data have been taken at $1,2,3$, and $4 \mathrm{~ms}$ after the discharge onset. The ${ }^{12} \mathrm{C}$ peaks are again normalized and the enrichment increase with time can be observed from the ${ }^{13} \mathrm{C}$ peaks. It can be seen that the rotating plasma did not achieve a quasi-equilibrium situation, suggesting that a longer vacuum chamber is necessary for the achievenent of a steady-state equilibrium.

Substituting $R=6 \mathrm{~cm}, \alpha=3.39$, and the value of $\omega=1.5 \times 10^{5} \mathrm{rad} / \mathrm{s}$ (measured for $B=0.12 \mathrm{~T}$ ) in Eq. (2), an ion temperature of $0.34 \mathrm{eV}$ is obtained. This value is in agreement with other results in arc experiments.

Further experiments must be done to determine an optimum value for the $B$ field, which maximizes the rotational velocity and therefore the enrichment. The high rotational 
velocities and separation factors presented here are consistent with the YALE centrifuge results and give an indication of the potential of the vacuum-arc centrifuge for isotope separation, as well as for the study of stable fully ionized rotating plasmas.

We express our thanks to Dr. M. Krishnan for his many suggestions and discussions during his visit to INPE's plasma laboratory. We would like to acknowledge discussions with Dr. A. Montes.
B. Bonnevier, Plasma Phys. 13, 763 (1971).

'B. W. James and S. W. Simpson, Phys. Lett. A 46, 347 (1974).

${ }^{3}$ H. Alfvén, Rev. Mod. Phys 32, 710 (1960).

${ }^{4}$ M. Krishnan, M. Geva, and J. L. Hirschfield, Phys. Rev. Lett. 46, 36 (1981).

'M. Krishnan, Phys. Fluids 26, 2676 (1983).

'E. Del Bosco, R. S. Dallaqua, and G. O. Ludwig, Rev. Bras. Apl. Vácuo 5, $160(1985)$.

M. Geva, M. Krishnan, and J. L. Hirshfield, J. Appl. Phys. 56, 1398 (1984). 